\title{
A importância da manutenção dos níveis de vitamina D para o sistema imunológico
}

\author{
The importance of maintaining vitamin D levels for the immune system \\ La importancia de mantener los niveles de vitamina D para el sistema inmunológico
}

Recebido: 10/09/2021 | Revisado: 16/09/2021 | Aceito: 20/09/2021 | Publicado: 21/09/2021

\author{
América Muraiare de Menezes \\ ORCID: https://orcid.org/0000-0002-1253-9391 \\ Centro Universitário Fametro, Brasil \\ E-mail: america_menezes@hotmail.com \\ Elizabeth Cidade dos Santos \\ ORCID: https://orcid.org/0000-0002-0205-1923 \\ Centro Universitário Fametro, Brasil \\ E-mail: eliz.beth.santos30@hotmail.com \\ Glaucilene da Conceição Otoni \\ ORCID: https://orcid.org/0000-0002-3924-2731 \\ Centro Universitário Fametro, Brasil \\ E-mail: glaucinhalindamao@gmail.com \\ José Carlos de Sales Ferreira \\ ORCID: https://orcid.org/0000-0002-1867-8229 \\ Centro Universitário Fametro, Brasil \\ E-mail: jcarlos.sales@gmail.com
}

\begin{abstract}
Resumo
Introdução: A vitamina D é um dos nutrientes em que se estabeleceu a estreita relação existente entre o seu status orgânico e o funcionamento do sistema Imune. Sua necessidade é suprida através da ingestão ou suplementação de alimentos como peixes gordurosos como salmão, atum, sardinha, cogumelos irradiados, dentre outros, sendo sua importância indiscutível para o organismo humano. Objetivo: Descrever a importância da vitamina D para o sistema imunológico, bem como sua atuação na prevenção de doenças autoimunes. Metodologia: Para a realização desse estudo optou-se por uma pesquisa na modalidade de revisão integrativa de literatura. Este método é apontado como ferramenta de grande relevância no campo da saúde, por proporcionar a busca, a avaliação crítica e a síntese de evidências sobre um tema investigado. Resultados e Discussão: Os aspectos nutricionais que cercam uma pessoa são extremamente importantes no equilíbrio do sistema imunológico, tendo um papel fundamental na manutenção da saúde do indivíduo, inclusive para portadores de doenças autoimunes. Conclusão: Ficou evidenciado através do desenvolvimento desse estudo que a vitamina D exerce um papel importante na regulação do sistema imunológico e, provavelmente, na prevenção das doenças imunomediadas.
\end{abstract}

Palavras-chave: Vitamina D; Doenças autoimune; Ação fisiológica.

\begin{abstract}
Introduction: Vitamin D is one of the nutrients in which the close relationship between its organic status and the functioning of the Immune system has been established. Its need is met through the ingestion or supplementation of foods such as fatty fish such as salmon, tuna, sardines, irradiated mushrooms, among others, and its importance for the human body is indisputable. Objective: To describe the importance of vitamin D for the immune system, as well as its role in preventing autoimmune diseases. Methodology: To carry out this study, we opted for a research in the modality of integrative literature review. This method is appointed as a tool of great relevance in the field of health, as it provides the search, critical assessment and synthesis of evidence on an investigated topic. Results and Discussion: The nutritional aspects that surround a person are extremely important in the balance of the immune system, playing a fundamental role in maintaining the individual's health, including for patients with autoimmune diseases. Conclusion: It was evident through the development of this study that vitamin D plays an important role in the regulation of the immune system and, probably, in the prevention of immune-mediated diseases.
\end{abstract}

Keywords: Vitamin D; Autoimmune diseases; Physiological action.

\section{Resumen}

Introducción: Se sabe que el sistema inmunológico consiste en una compleja red de células y moléculas encargadas de Introducción: La vitamina $\mathrm{D}$ es uno de los nutrientes en los que se ha establecido la estrecha relación entre su estado orgánico y el funcionamiento del sistema inmunológico. Su necesidad se satisface mediante la ingestión o suplementación de alimentos como pescados grasos como salmón, atún, sardinas, hongos irradiados, entre otros, y su importancia para el organismo humano es indiscutible. Objetivo: Describir la importancia de la vitamina D para el sistema inmunológico, así como su papel en la prevención de enfermedades autoinmunes. Metodología: Para la 
realización de este estudio se optó por una investigación en la modalidad de revisión integradora de la literatura. Este método es designado como una herramienta de gran relevancia en el campo de la salud, ya que proporciona la búsqueda, valoración crítica y síntesis de evidencia sobre un tema investigado. Resultados y Discusión: Los aspectos nutricionales que rodean a una persona son de suma importancia en el equilibrio del sistema inmunológico, jugando un papel fundamental en el mantenimiento de la salud del individuo, incluso para los pacientes con enfermedades autoinmunes. Conclusión: Se evidenció a través del desarrollo de este estudio que la vitamina D juega un papel importante en la regulación del sistema inmunológico y, probablemente, en la prevención de enfermedades inmunomediadas.

Palabras clave: Vitamina D; Enfermedades autoinmunes; Acción fisiológica.

\section{Introdução}

A importância da Vitamina D para o organismo é indiscutível, pois além de contribuir na homeostasia, previne também contra afecções de toda ordem. A síntese endógena da vitamina D inicia-se a partir da substância precursora, o 7 DHC, localizado na camada bi lipídica das membranas celulares das camadas profundas da epiderme. A absorção direta de luz solar, especificamente radiação ultravioleta B (UVB) nos comprimentos de onda entre 290 e 315 nanômetros, promove a fotólise, formando a pré-vitamina D3, que é termoestável e sofre uma reação de isomerização induzida pelo calor, formando a vitamina D3 (Oliveira, et al., 2014; Lucas, 2011).

A vitamina D é um dos nutrientes em que se estabeleceu a estreita relação existente entre seu status orgânico e o funcionamento do sistema imune. Diminuição de anticorpos humorais e da superfície de mucosas, da imunidade celular, da capacidade bactericida de fagócitos, da produção de complemento, do número total de linfócitos, do equilíbrio dos subtipos de linfócitos T. A vitamina em questão participa em várias ações dos sistemas imunológicos inato e adquirido (Brito, et al., 2017; Sassi, et al., 2018; Hewison, 2011).

Sua necessidade é suprida através de sua ingestão, (suplementação), alimentos como peixes gordurosos como salmão (selvagem), atum, sardinha, cogumelos irradiados, ou seja, que foram cultivados sob a luz do sol, leite integral e seus derivados (iogurte, manteiga, queijo), fígado de bovino, óleo de fígado de bacalhau e gema de ovo). A vitamina D pode ser obtida pelo organismo através dos alimentos sob a forma de colecalciferol ou vitamina D3 de origem animal, ou sob a forma de ergocalciferol ou vitamina D2 de origem vegetal (Catarino et al., 2016; Castro, 2011).

Casos de deficiência da vitamina D aumentaram em número de modo significante após a revolução industrial, mostrando que o estilo de vida das pessoas influência diretamente nas concentrações de vitamina D no organismo. Atualmente, tal deficiência é vista como um problema de saúde pública em todo o mundo, pois a deficiência de vitamina $\mathrm{D}$ pode levar à perda de densidade óssea, o que pode contribuir para a osteoporose e fraturas (ossos quebrados). Além disso, a deficiência severa também pode levar a outras doenças, como as autoimunes (Nieves, 2013; Wang, et al., 2017).

A interação da vitamina D com sistema imunológico vem sendo alvo de um número crescente de publicações nos últimos anos. Vários estudos tem relacionado a deficiência/insuficiência de vitamina D com diversas doenças autoimune incluindo Diabetes Mellitus (DM), Esclerose Múltipla (EM), Lúpus Eritematoso Sistêmico (LES), Doenças Inflamatórias Intestinais (DII) e Artrite Reumatoide (AR) (Agmon, et al., 2013).

No entanto, essa relação entre vitamina D e doenças autoimunes é pouco reconhecida em diagnósticos e tratamentos médicos, sendo essencial o desenvolvimento de mais pesquisas que possam esclarecer e promover a atuação desse micronutriente na prevenção e tratamento de doenças do sistema imunológico. O que remete a importância da presente pesquisa e da atuação cada vez mais presente do conhecimento do profissional de nutrição.

Desta forma, o objetivo geral desse estudo é descrever e relacionar a importância da vitamina D para o sistema imunológico e sua ação na prevenção e suporte no tratamento de doenças autoimunes. 


\section{Metodologia}

Para a realização desse estudo optou-se por uma pesquisa na modalidade de revisão integrativa de literatura. A revisão integrativa determina o conhecimento atual sobre uma temática específica, já que é conduzida de modo a identificar, analisar e sintetizar resultados de estudos independentes sobre o mesmo assunto (Silveira, et al., 2008).

A Revisão Integrativa é um método de pesquisa apontado como ferramenta de grande relevância no campo da saúde, por proporcionar a busca, a avaliação crítica e a síntese de evidências sobre um tema investigado. Esses aspectos facilitam a identificação dos resultados relevantes, de lacunas que direcionam para o desenvolvimento de futuras pesquisas e auxiliam o profissional a escolher condutas e a tomar decisões, proporcionando um saber crítico (Souza, Silva \& Carvalho, 2010).

Para extrair os dados dos artigos selecionados, será necessária a utilização de um instrumento previamente elaborado capaz de assegurar que a totalidade dos dados relevantes seja extraída, minimizar o risco de erros na transcrição, garantir precisão na checagem das informações e servir como registro (Souza, Silva \& Carvalho, 2010).

A coleta das informações para a pesquisa bibliográfica será por meio da exploração da base de dados da Biblioteca Virtual em Saúde (BVS), Biblioteca Cientifica Eletrônica Virtual (SCIELO) e Literatura Latino - Americana e do Caribe em Ciências da Saúde (LILACS).

A busca na base de dados será orientada pelos descritores: Vitamina D, doenças autoimunes, ação fisiológica; e será realizado em todos os índices, buscando captar o maior número de artigos publicados no período proposto que abordem a temática em discussão, visando o desenvolvimento fidedigno desse estudo.

Para uma análise crítica e reflexiva dos estudos incluídos na revisão, será realizada uma leitura minuciosa e criteriosa destacando os que atingirem os critérios de inclusão e que contemplarem o objetivo proposto, para viabilizar o resultado da pesquisa de forma objetiva.

Serão incluídos os artigos publicados em língua portuguesa nos últimos dez anos, com texto completo, disponível online, com acesso livre. Serão excluídos da amostra os artigos publicados em línguas estrangeiras, os que não apresentarem o texto na integra, artigos que não apresentavam relação direta com o tema, resumos, monografias, dissertações, teses e artigos repetidos.

\section{Resultados e Discussão}

\subsection{A vitamina D e sua relação com o sistema imunológico}

De acordo com Galvão et al., (2013) a vitamina D é um micronutriente que possui diversas funções no organismo, umas delas é promover a homeostase do cálcio e promover a saúde óssea. Em humanos, a vitamina D é obtida através da alimentação ou é sintetizada na pele, com a exposição ao sol. Ela atua no intestino, onde estimula a reabsorção do cálcio, e no osso, onde promove a diferenciação dos osteoblastos e a calcificação da matriz (Allgrove, 2015; Norman, 2012).

Além disso, a vitamina D tem vários efeitos nas células do sistema imunológico. Segundo Tortora e Derrickson (2016) esse sistema corresponde a um conjunto de células e mecanismo que atuam na defesa do corpo de organismos invasores estranhos, promovendo imunidade protetora. Por isso, é importante que a manutenção desse sistema seja realizada de forma adequada, com o consumo de nutrientes que ativam e renovam as células que compõe o sistema imunológico (Hewison, 2012; Basit, 2013).

Sendo assim, Rodrigues et al., (2019) descreve que é possível relacionar a ação da vitamina D e o mecanismo de defesa no corpo, pois uma das principais funções dela é ajudar a ativar as células T, também conhecidas como "células assassinas" no corpo, que compõe o grupo de células imunológicas. As células T realmente detectam e destroem patógenos estranhos, como vírus. Isso torna a vitamina D um nutriente essencial para a manutenção de um sistema imunológico em funcionamento, capaz de combater patógenos estranhos (Aranow, 2011; White, 2011). 
Sendo assim, Rodrigues et al., (2019) descreve que é possível relacionar a ação da vitamina D e o mecanismo de defesa no corpo, pois uma das principais funções dela é ajudar a ativar as células $\mathrm{T}$, também conhecidas como "células assassinas" no corpo, que compõe o grupo de células imunológicas. As células T realmente detectam e destroem patógenos estranhos, como vírus. Isso torna a vitamina D um nutriente essencial para a manutenção de um sistema imunológico em funcionamento, capaz de combater patógenos estranhos (Aranow, 2011; White, 2011).

Nesse contexto, entende-se que a deficiência de vitamina D pode contribuir para o aumento da autoimunidade e também ao aumento da suscetibilidade a infecções. Segundo Oliveira et al., (2014) essa deficiência pode ocorrer por falta de dieta, má absorção ou necessidade metabólica de quantidades maiores. Se a pessoa não ingerir vitamina D suficiente e não receber exposição suficiente ao sol ultravioleta por um longo período (consulte a seção acima) pode surgir uma deficiência. No entanto, quantidades acima do indicado podem ter efeitos negativos na saúde, como problemas renais, cálculos renais ou hipercalcemia, uma condição tóxica em que há muito cálcio no sangue (Chaves, 2014; Lichtenstein et al., 2013).

\subsection{A vitamina $D$ face a prevenção de doenças do sistema imunológico}

A vitamina D tem muitos efeitos no sistema imunológico. A deficiência de vitamina D está relacionada ao desenvolvimento de doenças autoimunes. Uma doença auto - imune ocorre quando o sistema imunológico ataca o corpo porque o confunde com algo estranho e existem muitas doenças autoimunes e suas causas muitas vezes podem estar associadas à deficiência de algum tipo de vitamina (Bitencourt \& Coann, 2019).

De acordo com Bellan, Pirisi e Sainaghi (2015) a deficiência de vitamina D está relacionada ao desenvolvimento e à gravidade da artrite reumatoide (AR) e causa inflamação e dor nas articulações. Acontece quando o sistema imunológico não funciona corretamente e ataca o revestimento das articulações (chamado de sinóvia). Em uma pessoa saudável, o sistema imunológico luta contra invasores, como bactérias e vírus. Com uma doença autoimune como a AR, o sistema imunológico confunde as células do corpo com invasores estranhos e libera substâncias químicas inflamatórias que atacam, no caso da AR. Essa doença se desenvolve em pacientes com deficiência de vitamina $\mathrm{D}$, e a atividade da doença está relacionada à deficiência de vitamina D (Pinheiro, 2015).

Segundo Simioni et al., (2016) a deficiência de vitamina D também está relacionada ao desenvolvimento de lúpus eritematoso sistêmico (LES). O LES se desenvolve em pacientes com deficiência de vitamina D, e a atividade da doença também é maior em pacientes com deficiência de vitamina D. O LES é uma doença crônica que pode ter fases de agravamento dos sintomas que se alternam com períodos de sintomas leves. A maioria das pessoas com LES consegue viver uma vida normal com o tratamento (Teixeira \& Costa, 2012).

A deficiência de vitamina D também está relacionada ao desenvolvimento e à gravidade da esclerose múltipla. Segundo Alves et al., (2014) a vitamina D deve ser administrada a pacientes com esclerose múltipla, e isso parece atenuar os sintomas da doença e prevenir a progressão da doença. A esclerose múltipla (EM) é uma condição que pode afetar o cérebro e a medula espinhal, causando uma ampla gama de sintomas potenciais, incluindo problemas de visão, movimentos de braços ou pernas, sensação ou equilíbrio (Pereira, 2020).

Os níveis ideais de vitamina $\mathrm{D}$ parecem ser necessários para a função imunológica normal e para a prevenção e tratamento de doenças autoimunes. Níveis baixos de vitamina D têm sido observados em pacientes com artrite reumatoide (AR), lúpus eritematoso sistêmico (LES) e avanço da esclerose múltipla. Além disso, de acordo com Delvin et al., (2014) a deficiência de vitamina $\mathrm{D}$ é um fator de risco para o desenvolvimento de algumas doenças autoimunes, como diabetes mellitus tipo 1, doença inflamatória intestinal e tireoidite de Hashimoto.

Além disso, alguns estudos apresentaram relação da vitamina D com a doença Vitiligo, que é um distúrbio pigmentar comum causado pela destruição de melanócitos funcionais. A vitamina D é um hormônio essencial sintetizado na pele e é 
responsável pela pigmentação da pele. Baixos níveis de vitamina $\mathrm{D}$ foram observados em pacientes com vitiligo e em pacientes com outras doenças autoimunes (Villegas, et al., 2019; Mendonça, et al., 2020).

Segundo Rosen et al., (2016) o desenvolvimento dessas doenças autoimune e a associação com a manifestação da deficiência de vitamina D pode ser explicado, pois a vitamina D é um imunomodulador potente. Têm efeitos diversos no sistema imunológico, ela potencializa a resposta imune inata aumentando a produção de macrófagos, monócitos e queratinócitos humanos, aumentando e potencializando a resposta imune contra patógenos externos e também afeta a resposta imune adaptativa (Oliveira \& Neto, 2021).

\subsection{Vitamina D e fontes de extração}

A vitamina D é uma vitamina solúvel em gordura que está naturalmente presente em alguns alimentos, adicionada a outros e disponível como suplemento dietético. Também é produzido endogenamente quando os raios ultravioleta (UV) da luz solar atingem a pele e ativa a síntese de vitamina D. A vitamina D obtida pela exposição ao sol, alimentos e suplementos é biologicamente inativa e deve passar por duas reações bioquímicas no corpo para sua ativação (Lichtenstein, et al., 2013; Galvão, et al., 2013).

A primeira reação é chamada de hidroxilação, ela ocorre no fígado e converte a vitamina $\mathrm{D}$ em 25-hidroxivitamina $\mathrm{D}$, também conhecida como "calcidiol". A segunda reação ocorre principalmente no rim e forma a 1,25-dihidroxivitamina D fisiologicamente ativa, também conhecida como "calcitriol". Sendo assim, para que a vitamina D exerça suas funções no organismo, ela precisa primeiramente ser consumida, depois sofrer reações bioquimicas com quebras de moléculas (Castro, 2011; Molina, 2021).

Além disso, a presença de vitamina D nos alimentos e suplementos dietéticos ocorre de duas formas principais, D2 (ergocalciferol) e D3 (colecalciferol), que diferem quimicamente apenas em suas estruturas. As duas formas são bem absorvidas no intestino delgado. A absorção ocorre de forma passiva e por um mecanismo que envolve proteínas transportadoras da membrana intestinal. A presença de gordura no intestino aumenta a absorção de vitamina $\mathrm{D}$, mas parte da vitamina D é absorvida mesmo sem gordura na dieta. Nem o envelhecimento nem a obesidade alteram a absorção de vitamina D pelo intestino (Castro, 2011; Molina, 2021).

Os níveis adequados de vitamina D para o consumo diário humano é recomendado e fornecido nas Dietary Reference Intakes (DRIs) desenvolvidas por comitês de especialistas. DRI é o termo geral para um conjunto de valores de referência usados para planejar e avaliar a ingestão de nutrientes por pessoas saudáveis, mas esses valores, que variam por idade e sexo (Amrein, 2020).

O consumo em excesso de vitamina D é tóxico, pois como ela aumenta a absorção de cálcio no trato gastrointestinal, a toxicidade da vitamina D resulta em hipercalcemia, que pode causar náuseas, vômitos, fraqueza muscular, distúrbios neuropsiquiátricos, dor, perda de apetite, desidratação, poliúria, sede excessiva e cálculos renais (Marins, et al., 2014).

\section{Considerações Finais}

Através do desenvolvimento desse estudo foi possível compreender a importância da vitamina D e sua influência no sistema imunológico, tendo em vista que que há uma evidente relação entre a deficiência de vitamina $\mathrm{D}$ e a prevalência de algumas doenças autoimunes que podem vir a comprometer esse sistema como um todo.

Nota-se ainda, que há a necessidade, no que se refere ao desenvolvimento de estudos relacionados a esta vertente, a fim de demonstrar e elucidar as mais diversas questões voltadas à temática abordada, tendo em vista que o número de pessoas que desenvolvem as doenças autoimunes vem aumentando exponencialmente. 


\section{Referências}

Agmon-Levin, N., et al. (2013). Vitamin D in systemic and organ-specific autoimmune diseases. Clinical reviews in allergy \& immunology, $45(2), 256-266$.

Allgrove, Jeremy. (2015). Physiology of calcium, phosphate, magnesium and vitamin D. Calcium and Bone Disorders in Children and Adolescents, 28 , 7-32.

Alves, B., et al. (2014). Esclerose múltipla: revisão dos principais tratamentos da doença. Saúde e meio ambiente: Revista interdisciplinar, 3(2), 19-34.

Alves, M., et al. (2013). Vitamina D-importância da avaliação laboratorial. Revista Portuguesa de Endocrinologia, Diabetes e Metabolismo, 8(1), 32-39.

Amrein, K., et al. (2020). Vitamin D deficiency 2.0: an update on the current status worldwide. European journal of clinical nutrition, 74(11), 1498-1513.

Aranow, C. (2011). Vitamin D and the immune system. Journal of investigative medicine, 59(6), 881-886.

Basit, S. (2013). Vitamin D in health and disease: a literature review. British journal of biomedical science, 70(4), 161-172.

Bellan, M., Pirisi, M., \& Sainaghi, P. P. (2015). Osteoporose na artrite reumatoide: papel do sistema vitamina D/hormônio paratireóideo. Revista Brasileira de Reumatologia, 55(3), 256-263.

Bitencourt, R. M., \& Coan, F. C. (2019). O uso da vitamina d em doenças autoimunes: revisão sobre o potencial terapêutico. Inova Saúde, 9(1), 12-33.

Bouillon, R. (2017). Comparative analysis of nutritional guidelines for vitamin D. Nature Reviews Endocrinology. 13, 466-479.

Castro, Luiz Claudio Gonçalves de. (2011). O sistema endocrinológico vitamina D. Arquivos Brasileiros de Endocrinologia \& Metabologia, 55(8), 566-575.

Chaves, K. L. L., Maia, F. A., \& Almeida, M. T. C. (2014). Efeitos da deficiência e do excesso de vitaminas no organismo. Anais do VIII Fórum FEPEG, 2427.

De Oliveira, V., et al. (2014). Influência da vitamina D na saúde humana. Acta bioquím. clín. latinoam, 339-347.

Delvin, E., et al. (2014). Role of vitamin D in acquired immune and autoimmune diseases. Critical reviews in clinical laboratory sciences, $51(4), 232-247$.

Freire, R., Ingano, L., Serena, G., Cetinbas, M., Anselmo, A., Sapone, A., et al. (2019). Human gut derived-organoids provide model to study gluten response and effects of microbiota derived molecules in celiac disease. Nature. 9(7029), 1-15.

Gerhardt, T. E., Pinheiro, R., Ruiz, E. N. F., Silva Junior, A. G. (2016). Itinerários terapêuticos: integralidade no cuidado, avaliação e formação em saúde Rio de Janeiro: CPESC. 13-26.

Galvão, L. B., et al. (2013). Considerações atuais sobre a vitamina D. Brasília Med, 50(4), 324-332.

Hewison, Martin. (2012). An update on vitamin D and human immunity. Clinical endocrinology, 76(3), 315-325.

Hewison, Martin. (2011). Antibacterial effects of vitamin D. Nature Reviews Endocrinology, 7(6), 337-345.

Lichtenstein, A., et al. (2013). Vitamina D: ações extraósseas e uso racional. Revista da Associação Médica Brasileira, 59(5), 495-506.

Liang, Y., Meng, F. Y., Pan, H. F., \& Ye, D. Q. (2015). A Literature Review on Patients with Autoimmune Diseases Following Vaccination Against Infections, Human Vaccines and Immunotherapeutics, 11(9), 2274-2280.

Löw, I. (2018). Tangled diagnoses: Prenatal Testing, Women, and Risk Chicago: The University of Chicago Press.

Luz, G. S., Silva, M. R. S., \& Demontigny, F. (2016). Necessidades prioritárias referidas pelas famílias de pessoas com doenças raras. Texto \& Contexto Enfermagem, 25(4):1-9.

Lucas, Ana Catarina Gonçalves. (2011). Importância da vitamina D no estado de saúde das populações. Tese de Doutorado.

Marins, T. A., et al. (2014). Intoxicação por vitamina D: relato de caso. Einstein (São Paulo), 12(2), $242-244$.

Mendonca, A. E. A., et al. (2020). Aspectos sobre a etiopatogênese e terapêutica do vitiligo. Revista de Medicina, 99(3), 278-285.

Mehta, A, et al. (2017). Exploring the patient journey to diagnosis of Gaucher disease from the perspective of 212 patients with Gaucher disease and 16 Gaucher expert physicians. Molecular Genetics and Metabolism 122(3), 122-129.

Mills, J. R., Murray J. A. (2016). Contemporary celiac disease diagnosis: is a biopsy avoidable? Curr Opin Gastroenterol. 32, 80-85.

Molina, Patricia E. (2021). Fisiologia Endócrina-5. McGraw Hill Brasil.

Mohammed, G. F., Gomaa, A. H., \& Al-Dhubaibi, M. S. (2015). Highlights in Pathogenesis of Vitiligo, World Journal of Clinical Cases, 3(3), 221-230.

Mohammdibakhsh, R., Sohrabi, R., Salemi, M., Mirghaed, M. T., \& Behzadifar, M. Celiac disease in Iran: a systematic review and meta-analysis. Electron Physician. 2017;9(3):3883-3895.

Murdaca, G., et al. (2019). Emerging role of vitamin D in autoimmune diseases: An update on evidence and therapeutic implications. Autoimmunity reviews, 18(9), 102350.

Nieves, J. W. (2013). Skeletal effects of nutrients and nutraceuticals, beyond calcium and vitamin D. Osteoporosis International, 24,3 , 771-786. 
Norman, Anthony. (2012). Vitamin D. Elsevier.

Oliveira, K. J. J., \& Neto, J. R. T. V. (2021). A inserção de vitamina d no tratamento de doenças autoimunes. Revista Multidisciplinar em Saúde, 2(2), 58-58.

Padovani, R. M., et al. (20016). Dietary reference intakes: aplicabilidade das tabelas em estudos nutricionais. Revista de Nutrição, 19(6), 741-760.

Pereira, Ana Rita Rodrigues. (2020). Relatório de Estágio e Monografia intitulada "Mecanismos Moduladores da Vitamina D na Esclerose Múltipla. Tese de Doutorado. Universidade de Coimbra.

Pinheiro, Joana. (2015). Terapêutica Nutricional na Artrite Reumatóide. Acta Portuguesa de Nutrição, 3, 26-30.

Prodanov, C. C., \& De Freitas, E. C. (2013). Metodologia do trabalho científico: métodos e técnicas da pesquisa e do trabalho acadêmico-2a Edição. Editora Feevale, 51-52.

Rodrigues, B. B., et al. (2019). Vitamina D na regulação do organismo humano e implicações de sua deficiência corporal. Brazilian Journal of Health Review, $2(5), 4682-4692$.

Rosen, Y., et al. (2016). Vitamin D and autoimmunity. Scandinavian journal of rheumatology, 45(6), 439-447.

Ross, A. (2011). Catharine et al. The 2011 report on dietary reference intakes for calcium and vitamin D from the Institute of Medicine: what clinicians need to know. The Journal of Clinical Endocrinology \& Metabolism, 96(1),53-58.

Sahota, Opinder. (2018). Understanding vitamin D deficiency. Age and ageing, 43(5), 589, 2014. Sassi, F.; Tamone, C.; D'amelio, P.. Vitamin D: nutrient, hormone, and immunomodulator. Nutrients, 10(11), 1656.

Simioni, J. A., Heimovski, F., SkarE, T. L. (2016). Acerca de lúpus, vitamina D e leucopenia. Revista brasileira de reumatologia, 56(3), $206-211$.

Souza, M. T., Silva, M. D., \& Carvalho, R. (2010). Revisão integrativa: o que é e como 15 fazer. Einstein, 8(1), 102-106.

Sorice, A., Guerriero E., Capone F., et al. (2014). Ascorbic acid: its role in immune system and chronic inflammation diseases. Mini-Reviews in Medicinal Chemistry, 14(5), 444-52.

Sun, X., Lu L., Yang, R., Li, Y., Shan, L., \& Wang, Y. (2016). Increased incidence of thyroid disease in patients with celiac disease: a systematic review and meta-analysis. Plos One. 11(12)

Teixeira, T. M., Costa, C. L. da. (2012). Papel da vitamina D nos lúpus eritematoso sistêmico. Revista de Nutrição, 25(4),531-538.

Tortora, G. J., \& Derrickson, Bryan. (2019). Corpo Humano-: Fundamentos de Anatomia e Fisiologia. Artmed Editora, 2016. Villegas, A. C. et al. Vitiligo y vitamina D. Revista de la Asociación Colombiana de Dermatología y Cirugía Dermatológica, 27(1), 36-51.

Wang, H., et al. (2017). Vitamin D and chronic diseases. Aging and disease, 8(3), 346.

White, John H. (2012). Vitamin D metabolism and signaling in the immune system. Reviews in endocrine and metabolic disorders, 13(1), 21-29.

Zaahur, M., \& ASIF, A. R. (2013). Clinical, Cellular and Molecular Biology of autoimmune Disorders - Introduction, Journal of Clinical and Cellular Immunology, 1-2.

Zhao, Z., Zou, J., Zhao, L., Cheng, Y., Cai, H., Li, M., et al. (2016). Celiac disease autoimmunity in patients with autoimmune diabetes and thyroid disease among Chinese population. Plos One. 11(7). 